Revista Geográfica Digital. IGUNNE. Facultad de Humanidades. UNNE. Año 11. № 21.

Enero - Junio 2014. ISSN 1668-5180 Resistencia, Chaco

\title{
APLICACIONES DIDACTICAS CON SISTEMAS DE INFORMACIÓN GEOGRÁFICA. UNA APROXIMACIÓN AL CLIMA Y EL PAISAJE RESULTANTE EN EL CONTINENTE AFRICANO
}

\section{Resumen}

Mgtr. Vilma Lilian Falcón

vfalcon 1609@hotmail.com

Jefe de Trabajos Prácticos

Geografía de Africa y Oceanía

Facultad de Humanidades-UNNE

Las experiencias pedagógicas en el aula brindan continuamente enormes posibilidades de plasmar por escrito no solo el resultado al que se llega con cada propuesta de trabajo sino también del proceso que ello implica. Este artículo refleja uno de esos casos ya que pretende describir una experiencia didáctica, llevada a cabo en una cátedra, basada en conceptos y contenidos teóricos referidos a las condiciones del medio natural en África, utilizando los recursos tecnológicos que proveen los Sistemas de Información Geográfica (SIG).

\section{Introducción}

La experiencia particular a la que nos referimos en esta presentación se enmarca en el desarrollo de los trabajos prácticos correspondientes a la cátedra Geografía de África y Oceanía, espacio curricular que conforma el plan de estudio del profesorado y licenciatura en Geografía de la Facultad de Humanidades de la UNNE. Como se señala en el Plan de la cátedra desde la cual se expone esta experiencia, uno de los objetivos primordiales de los espacios teóricos y prácticos es ofrecer al estudiante las herramientas metodológicas y técnicas aplicables al desarrollo de pequeñas investigaciones que le permitan, en primer lugar, analizar una problemática específica con criterio propio; en segundo lugar, desarrollar un sentido crítico frente a las teorías, las lecturas y los contenidos provistos por la Cátedra y, finalmente, entrenarse aplicando técnicas e instrumentos en casos de estudio.

En esta oportunidad nos propusimos seguir algunos de los procedimientos planteados por Bruniard Enrique (1992) en su obra Climatología, procesos y tipos climáticos, quien señala que utilizando solo datos térmicos y pluviométricos -en este caso de localidades del continente africano- se puede localizar, describir y explicar el tipo climático correspondiente y las condiciones fisiográficas generales del ámbito en la que se encuentra. Específicamente hemos adoptado una serie de pasos que incluyen

Publicado en formato digital: Mgtr. Vilma Lilian Falcón. APLICACIONES DIDACTICAS CON SISTEMAS DE INFORMACIÓN GEOGRÁFICA. UNA APROXIMACIÓN AL CLIMA Y EL PAISAJE RESULTANTE EN EL CONTINENTE AFRICANO. Resúmenes. Revista Geográfica Digital. IGUNNE. Facultad de Humanidades. UNNE. Año 11. № 21. Enero - Junio 2014. ISSN 1668-5180 Resistencia, Chaco.

En: http://hum.unne.edu.ar/revistas/geoweb/default.htm 
Revista Geográfica Digital. IGUNNE. Facultad de Humanidades. UNNE. Año 11. № 21.

Enero - Junio 2014. ISSN 1668-5180 Resistencia, Chaco

cálculos sencillos y análisis simples de los datos termo-pluviométricos apoyados en algunas de las herramientas que provee ArcGis.

Conviene señalar que los objetivos específicos que se persiguen con esta aplicación práctica apuntan a: favorecer la comprensión de los temas teóricos referidos a las condiciones climáticas del continente africano; propiciar el uso de saberes previos en el proceso de aprendizaje de nuevos contenidos teóricos y prácticos; integrar los conceptos específicos de la disciplina geográfica y de las ciencias conexas sobre las que se basan las técnicas a aplicar con los conceptos propios que se manejan en el campo de los SIG y, finalmente, fortalecer el uso de herramientas y técnicas aplicables desde los Sistemas de Información Geográfica.

\section{Los conceptos y contenidos teóricos en cuestión}

La propuesta de este trabajo se enmarca en el análisis que propone la Unidad 3: el clima de África en el contexto del eje: El Medio Ambiente Físico y, en ese sentido, pretende contribuir a dar respuesta a una pregunta que surge en el proceso de enseñanza-aprendizaje y que se sintetiza del siguiente modo: ¿cómo se manifiestan los componentes del medio natural en el continente africano?.

Para introducirnos al tema y brindar un marco general de análisis recurrimos a un párrafo de Méndez R. y F. Molinero (2002:578) en el que puede leerse la siguiente caracterización que realizan los autores para el continente africano: "los climas son los verdaderos dueños de la tierra africana $\lceil$..... Africa es el continente cálido por excelencia...salvo el sector septentrional del Maghreb, el mediterráneo del El Cabo y una estrecha franja en el SE costero del hemisferio austral, está situada en la zona cálida entendiendo por tal aquella en que la temperatura media del mes más frío no desciende de los $18^{\circ} \mathrm{C}$ según el criterio de Köppen". Por su parte, Bruniard Enrique (1992) señala por ejemplo que "en Africa, las isohietas y las isotermas tienen un trazado casi zonal con marcado paralelismo" y que las temperaturas alcanzan sus mayores valores en el desierto sahariano, mientras que al sur del Ecuador, en las zonas costeras reciben la influencia moderadora del mar. Precisamente lo que intentamos llevar a la práctica es la propuesta de análisis que realiza este último autor ya que resulta un modo sencillo de aproximarse a las particularidades del paisaje del espacio en estudio, a la vez que permite comprender cuales son los elementos básicos que interactúan en su determinación.

Publicado en formato digital: Mgtr. Vilma Lilian Falcón. APLICACIONES DIDACTICAS CON SISTEMAS DE INFORMACIÓN GEOGRÁFICA. UNA APROXIMACIÓN AL CLIMA Y EL PAISAJE RESULTANTE EN EL CONTINENTE AFRICANO. Resúmenes. Revista Geográfica Digital. IGUNNE. Facultad de Humanidades. UNNE. Año 11. № 21. Enero - Junio 2014. ISSN 1668-5180 Resistencia, Chaco.

En: http://hum.unne.edu.ar/revistas/geoweb/default.htm 
Revista Geográfica Digital. IGUNNE. Facultad de Humanidades. UNNE. Año 11. № 21.

Enero - Junio 2014. ISSN 1668-5180 Resistencia, Chaco

Sin dudas, conceptos como clima, aridez, humedad, paisaje natural, evapotranspiración, propios de la disciplina geográfica, se integran con otros de las ciencias conexas sobre las que se basan las técnicas a aplicar: geoprocesamiento, datos vectoriales, datos raster, atributos, interpolación, isopletas; éstos son solo algunos ejemplos de los que aparecen en el proceso de desarrollo del trabajo. Como lo señala Zapettini, M.C.(2007:194), en una propuesta educativa, el éxito en la utilización de un SIG no está dado simplemente por el tipo de SIG o por el software elegido; Io importante es la metodología general implementada desde la identificación de un problema, la evaluación de la calidad de los datos, su integración, los métodos y procesos aplicados para producir la nueva información y evaluar los resultados útiles.

\section{Los recursos necesarios}

\section{Los datos de partida}

Los datos estadísticos de cada localidad se constituyen en el insumo principal a partir del cual el alumno puede realizar el proceso de análisis y obtener las representaciones gráficas y cartográficas, elementos que ayudan a la comprensión de las particularidades de los componentes físicos desarrollados con mayores detalles en las clases teóricas. En esta propuesta nos apoyaremos en los resultados obtenidos y comentados en un trabajo previo de similares características (Falcón, V. y Da Silva, C. 2013) en la que el principal producto residía en la geolocalización de más de 550 puntos que representan estaciones meteorológicas de África y Oceanía con datos de índole físico (temperatura, precipitación, altura sobre el nivel del mar obtenidos de fuentes analógicas y cuyo procesamiento permitió contar con información digital. Con base en dicha información se propuso realizar el procesamiento de los datos primarios para obtener valores derivados como por ejemplo las diferencias térmicas de los meses extremos de verano e invierno y que suelen utilizarse como indicativos del grado de continentalidad; valores combinados, como resultado de la asociación de los datos térmicos y pluviométricos como ser el Índice de Aridez y cuya aplicación se explica más adelante.

\section{El aporte de los SIG}

Hemos recurrido a ArcGIS Desktop de la empresa ESRI (Environmental Systems Research Institute) que posee un conjunto de aplicaciones integradas a través de sus compentes principales: ArcMap - ArcCatlog y ArcToolbox. Entre los módulos requeridos para lograr nuestro objetivo hemos recurrido al Spatial Analys Tool y al 3D Analys Tools con sus herramientas para generar mapas raster e isolíneas a partir de

Publicado en formato digital: Mgtr. Vilma Lilian Falcón. APLICACIONES DIDACTICAS CON SISTEMAS DE INFORMACIÓN GEOGRÁFICA. UNA APROXIMACIÓN AL CLIMA Y EL PAISAJE RESULTANTE EN EL CONTINENTE AFRICANO. Resúmenes. Revista Geográfica Digital. IGUNNE. Facultad de Humanidades. UNNE. Año 11. № 21. Enero - Junio 2014. ISSN 1668-5180 Resistencia, Chaco.

En: http://hum.unne.edu.ar/revistas/geoweb/default.htm 
Revista Geográfica Digital. IGUNNE. Facultad de Humanidades. UNNE. Año 11. № 21.

Enero - Junio 2014. ISSN 1668-5180 Resistencia, Chaco

un archivo de puntos. De modo sintético exponemos el procedimiento necesarios para obtener un shapefile de isolíneas utilizando el ArcGIS ${ }^{1}$ :

1. Contar con el shape de puntos que contengan la variable en estudio (precipitación media anual, temperatura, índice de aridez) del Espacio estudiado. Se debe adicionar el shape del contorno de cada continente ya que se necesitará para efectuar el recorte del raster obtenido.

2. Generar el raster con los valores de la variable que se pretende representar usando el método de interpolación más apropiado (en este caso IDW). Este paso se logra con el módulo Spatial Analys Tool desplegado desde ArcToolbox, usando la herramienta Interpolación.

3. Crear las isolíneas utilizando el raster anterior, para lo cual se utilizará la herramienta countor list desde la aplicación Raster Surface del módulo 3D Analyst Tool.

4. Para recortar el shape de isolíneas con respecto a la región en estudio se utiliza la herramienta clip desde el módulo Geoprocessing cuando se trata de un shape vectorial (líneas) y desde Extract By Mask del Spatial Analys Tool cuando se trata de un formato raster.

\section{Aplicaciones para el Geoproceso: Interpolación}

Al trabajar con un conjunto limitado de datos en espacios geográficos de índole continental, necesariamente se debe recurrir a la técnica de interpolación. En estadística, la interpolación es usada para estimar valores desconocidos a partir de una serie de datos conocidos y ponderados (Murillo et. al., 2012). Desde un punto de vista general, los métodos de interpolación se clasifican en dos grandes grupos: métodos globales y métodos locales determinísticos. Los métodos globales utilizan todo los datos disponibles para efectuar una estimación válida para toda la región de interés; en cambio los métodos locales determinísticos operan dentro de una pequeña zona alrededor de la ubicación donde se desea obtener un valor interpolado. (Cfr. González Zumba, 2006).

En el caso particular de esta propuesta se utilizó el método el IDW (Inverse Distance Weighting) para obtener la cartografía temática de los datos termopluviométricos y del Índice de Aridez calculado en el proceso. Este método utiliza una técnica de interpolación para estimar los valores de las celdas calculando promedios de

\footnotetext{
${ }^{1}$ Es necesario destacar que en la práctica el alumno cuenta, además de la guía de Trabajo Práctico, con un Anexo de tipo tutorial; se trata de un documento en el que se expone el procedimiento a seguir, las herramientas a utilizar y las imágenes capturadas en la pantalla que permiten observar cada instancia de las tareas a realizar con las herramientas que provee ArcGis.
}

Publicado en formato digital: Mgtr. Vilma Lilian Falcón. APLICACIONES DIDACTICAS CON SISTEMAS DE INFORMACIÓN GEOGRÁFICA. UNA APROXIMACIÓN AL CLIMA Y EL PAISAJE RESULTANTE EN EL CONTINENTE AFRICANO. Resúmenes. Revista Geográfica Digital. IGUNNE. Facultad de Humanidades. UNNE. Año 11. № 21. Enero - Junio 2014. ISSN 1668-5180 Resistencia, Chaco.

En: http://hum.unne.edu.ar/revistas/geoweb/default.htm 
Revista Geográfica Digital. IGUNNE. Facultad de Humanidades. UNNE. Año 11. № 21.

Enero - Junio 2014. ISSN 1668-5180 Resistencia, Chaco

los valores de los puntos de datos de muestra en la vecindad de cada celda de procesamiento. Este método plantea que cuanto más cerca está un punto del centro de la celda que se está estimando, más influencia o peso tendrá en el proceso de cálculo del promedio.

\section{Análisis y elaboración de cartografía temática}

Señalábamos que los datos de partida con que cuenta el alumno son: Temperaturas y precipitaciones medias mensuales y anuales. Sin olvidar la pregunta que fundamenta esta práctica, la propuesta se orienta hacia dos vertientes: un análisis simple de los datos térmico-pluviométrico que le permitirá ensayar respuestas y conclusiones solo con visualizar en pantalla los resultados del análisis y otro más complejo que surgirá de la aplicación de fórmula matemática y obtención de resultado como insumo para una salida cartográfica.

Bruniard, E. (1992) destaca la importancia de conocer los valores de temperatura que resultan significativos para producir cambios entre los diferentes paisajes naturales y por ello reconoce los límites térmicos propuestos en los diversos sistemas climatológicos definidos según el criterio de los autores: SUPAN (1880), THORNTHWAITE (1931 y1948), KÖPEEN (1930), MILLER (1975) entre otros. Estos últimos coinciden en considerar a la isoterma de $18^{\circ} \mathrm{C}$ como límite para definir los climas cálidos y el valor de $6^{\circ} \mathrm{C}$ como valor mínimo necesario para la actividad vegetal.

Entonces, como primera aplicación y siempre teniendo en cuenta la utilidad de la información resultante para la comprensión del aspecto o variable analizado en el espacio estudiado, hemos incorporado algunos procedimientos que se encuadran en los criterios adoptados por Köppen y Miller para lograr una aproximación a la asociación entre distribución geográfica de la temperatura y la humedad y los paisajes naturales resultantes.

Para el primer caso la propuesta es observar cómo se distribuye la temperatura a partir del trazado de las isotermas; para ello tomamos como referencia los valores propuestos por ambos autores para establecer los límites térmicos en su clasificación climática.

Dada la particularidad de los climas en el continente africano se propuso observar especialmente el trazado de la isoterma de $18^{\circ} \mathrm{C}$ dado que este valor representaría el límite de los climas cálidos y la capacidad de actividad vegetativa por lo que, en forma indirecta, estaría indicando la distribución geográfica de las grandes formaciones vegetales. (Cfr. Bruniard, E.1992: 42-48)

Publicado en formato digital: Mgtr. Vilma Lilian Falcón. APLICACIONES DIDACTICAS CON SISTEMAS DE INFORMACIÓN GEOGRÁFICA. UNA APROXIMACIÓN AL CLIMA Y EL PAISAJE RESULTANTE EN EL CONTINENTE AFRICANO. Resúmenes. Revista Geográfica Digital. IGUNNE. Facultad de Humanidades. UNNE. Año 11. № 21. Enero - Junio 2014. ISSN 1668-5180 Resistencia, Chaco.

En: http://hum.unne.edu.ar/revistas/geoweb/default.htm 
Revista Geográfica Digital. IGUNNE. Facultad de Humanidades. UNNE. Año 11. № 21.

Enero - Junio 2014. ISSN 1668-5180 Resistencia, Chaco

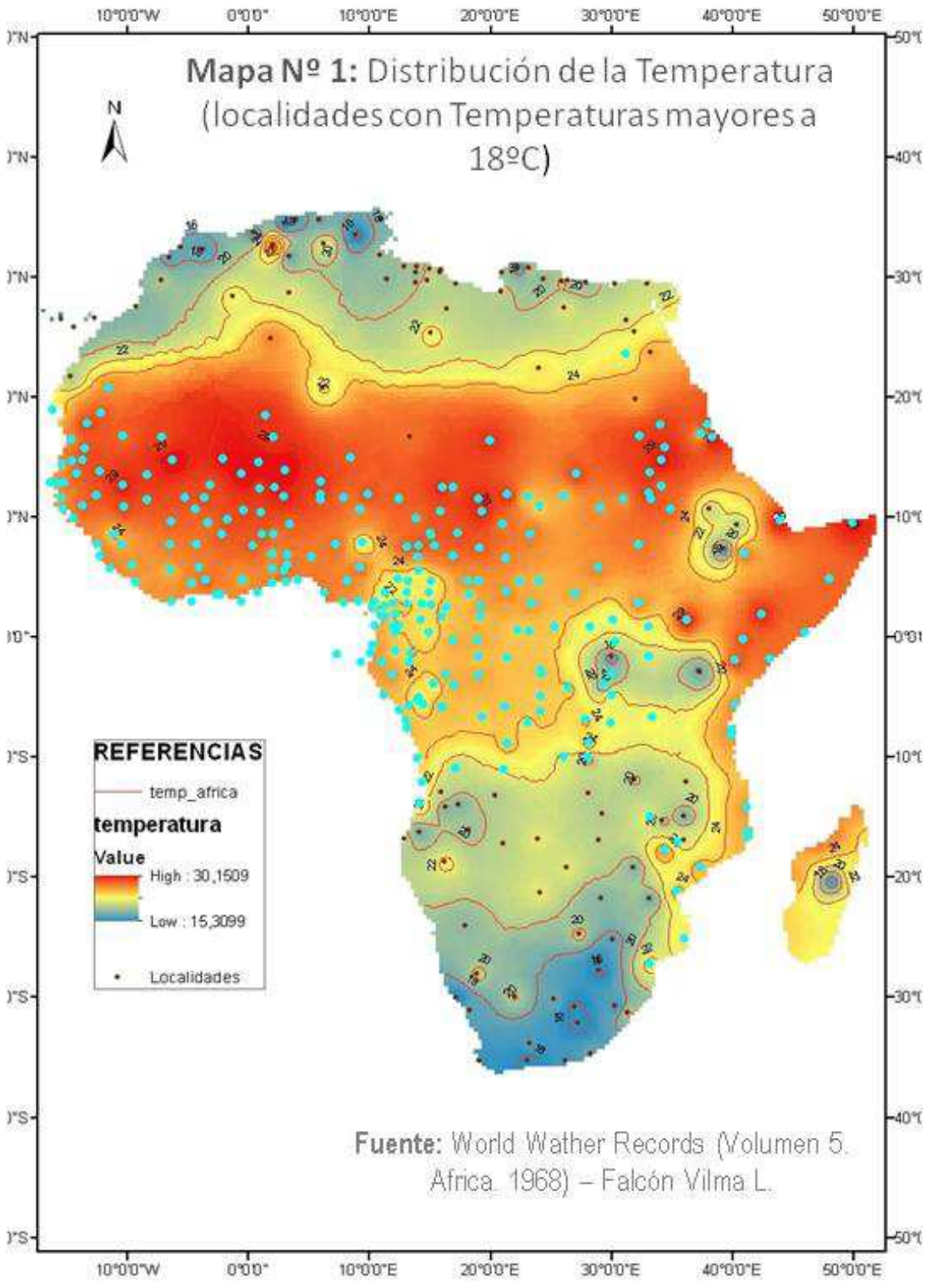

Como parte del análisis se seleccionaron las localidades que presentan temperaturas mayores a $18^{\circ} \mathrm{C}$ en todos los meses del año, así se puede advertir (Mapa № 1) la disposición zonal del "calor constante" con ausencia de heladas y sin período de reposo vegetativo.

De la misma manera se procedió para determinar los espacios asociados a climas templados cálidos o subtropicales, por lo que se seleccionaron las localidades que no presentan ningún mes con temperatura inferior a $6^{\circ} \mathrm{C}$.

En el mapa № 2 se muestra el resultado de la búsqueda, advirtiéndose que quedan seleccionadas todas las localidades, confirmando así la idea inicial sobre la que se trabajó al desarrollar esta propuesta cuando se expresa que "África es el continente cálido por excelencia y salvo el sector septentrional del Maghreb, el mediterráneo del El Cabo y una estrecha franja en el SE costero del hemisferio austral, está situada en la zona cálida"

Publicado en formato digital: Mgtr. Vilma Lilian Falcón. APLICACIONES DIDACTICAS CON SISTEMAS DE INFORMACIÓN GEOGRÁFICA. UNA APROXIMACIÓN AL CLIMA Y EL PAISAJE RESULTANTE EN EL CONTINENTE AFRICANO. Resúmenes. Revista Geográfica Digital. IGUNNE. Facultad de Humanidades. UNNE. Año 11. № 21. Enero - Junio 2014. ISSN 1668-5180 Resistencia, Chaco.

En: http://hum.unne.edu.ar/revistas/geoweb/default.htm 
Revista Geográfica Digital. IGUNNE. Facultad de Humanidades. UNNE. Año 11. № 21. Enero - Junio 2014. ISSN 1668-5180 Resistencia, Chaco

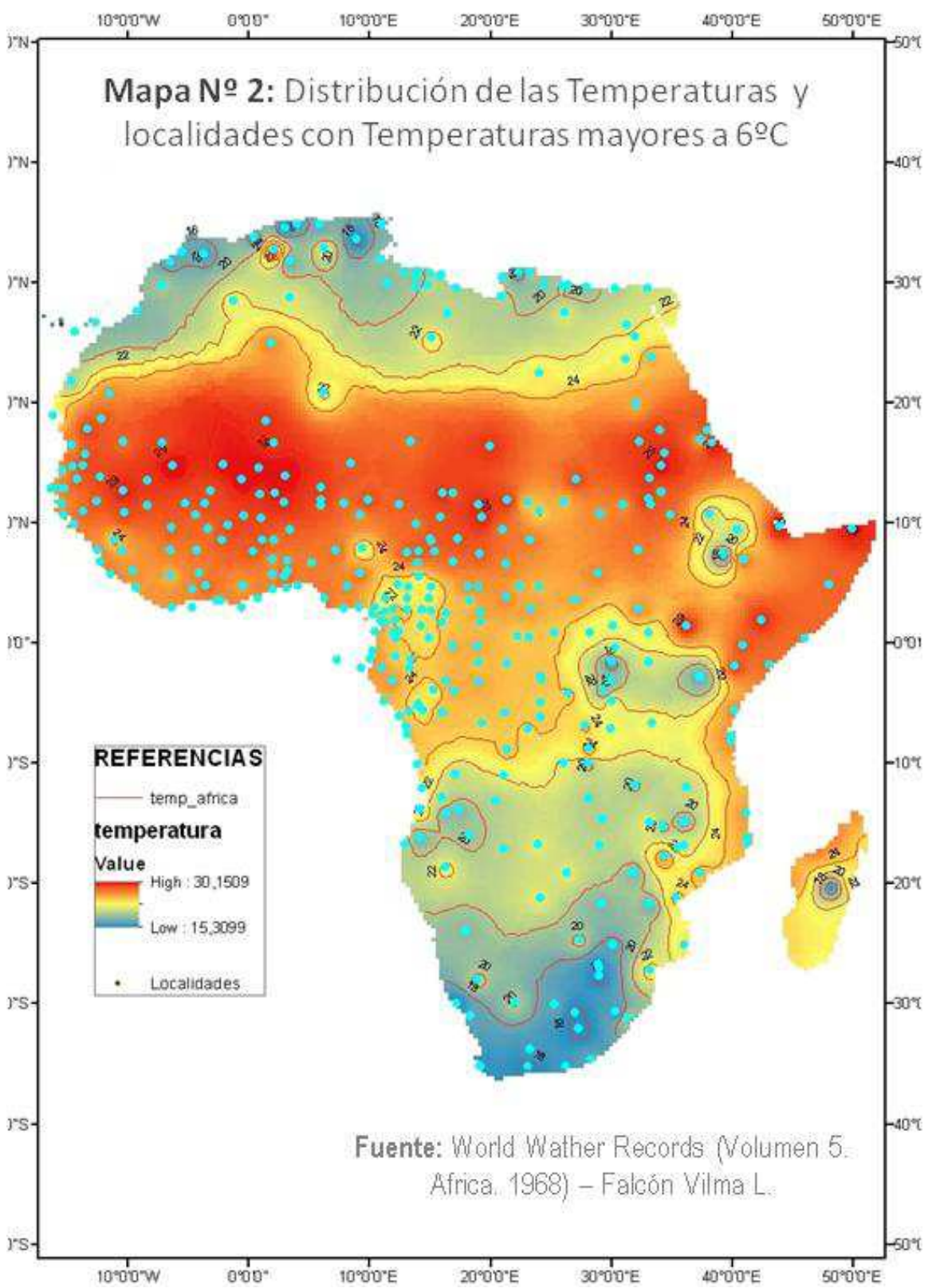

Siguiendo la propuesta de Bruniard E. $(1992: 36,44)$ quien señala que el Factor Humedad es la primera consideración que ha de realizarse para observar la asociación entre ésta y la distribución de la vegetación natural, incorporamos al trabajo la representación de la distribución de precipitaciones medias anuales (Mapa № 3).

Publicado en formato digital: Mgtr. Vilma Lilian Falcón. APLICACIONES DIDACTICAS CON SISTEMAS DE INFORMACIÓN GEOGRÁFICA. UNA APROXIMACIÓN AL CLIMA Y EL PAISAJE RESULTANTE EN EL CONTINENTE AFRICANO. Resúmenes. Revista Geográfica Digital. IGUNNE. Facultad de Humanidades. UNNE. Año 11. № 21. Enero - Junio 2014. ISSN 1668-5180 Resistencia, Chaco.

En: http://hum.unne.edu.ar/revistas/geoweb/default.htm 
Revista Geográfica Digital. IGUNNE. Facultad de Humanidades. UNNE. Año 11. № 21. Enero - Junio 2014. ISSN 1668-5180 Resistencia, Chaco

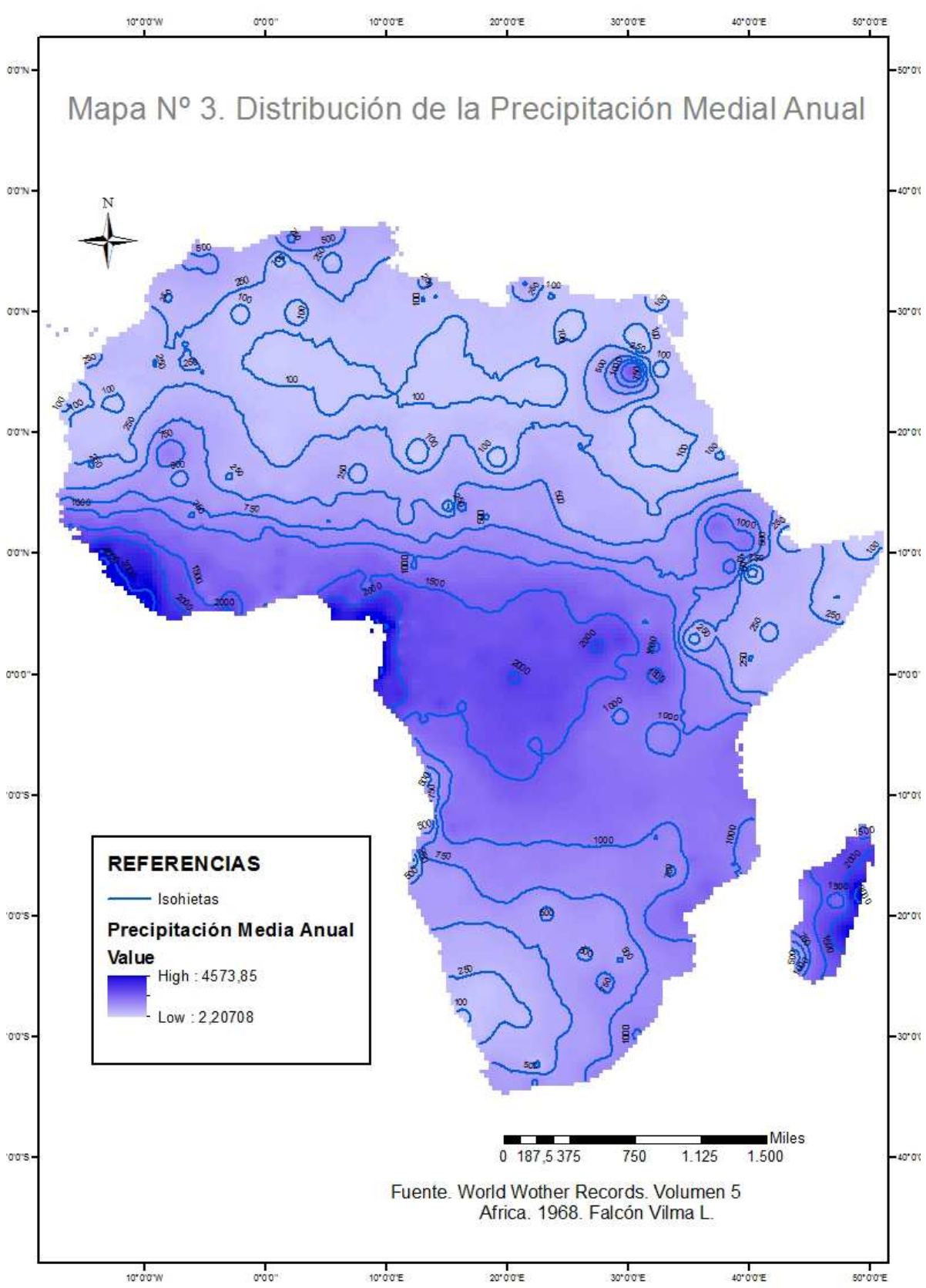

\section{El Indice de Aridez}

Teniendo en cuenta lo analizado anteriormente nos hemos propuesto obtener el Índice de Aridez según la fórmula ideada por De Martonne en 1926. Este índice (IA) relaciona la lámina de agua caída durante el año (precipitación en $\mathrm{mm}$ ) con la temperatura media anual (Temperatura en ${ }^{\circ} \mathrm{C}$ ) a la que se le adiciona el valor de 10 como constante. El resultado de dicha combinación expresa "en sus grandes trazos el balance hídrico anual entre las entradas, representadas por las precipitaciones y las

Publicado en formato digital: Mgtr. Vilma Lilian Falcón. APLICACIONES DIDACTICAS CON SISTEMAS DE INFORMACIÓN GEOGRÁFICA. UNA APROXIMACIÓN AL CLIMA Y EL PAISAJE RESULTANTE EN EL CONTINENTE AFRICANO. Resúmenes. Revista Geográfica Digital. IGUNNE. Facultad de Humanidades. UNNE. Año 11. № 21. Enero - Junio 2014. ISSN 1668-5180 Resistencia, Chaco.

En: http://hum.unne.edu.ar/revistas/geoweb/default.htm 
salidas o pérdidas generadas por la evapotranspiración como una consecuencia de la temperatura" (cfr. Bruniard, E. 1992:38).

Tomando como base los datos de temperatura y precipitación de las estaciones meteorológicas de África con que se viene trabajando, el alumno puede calcular el Índice de Aridez en la misma tabla de atributos en forma rápida, aplicando la siguiente fórmula y utilizando las herramientas de cálculo que ofrece ArcGis:

$$
\mathrm{IA}=\frac{\mathrm{P}}{\mathrm{T}+10}
$$

Una vez obtenido el dato para cada localidad, se procede de la misma manera que en las anteriores representaciones para lograr la cartografía correspondiente, de modo que el mapa del Índice de Aridez reflejará un resultado similar al que se muestra en el mapa № 4.

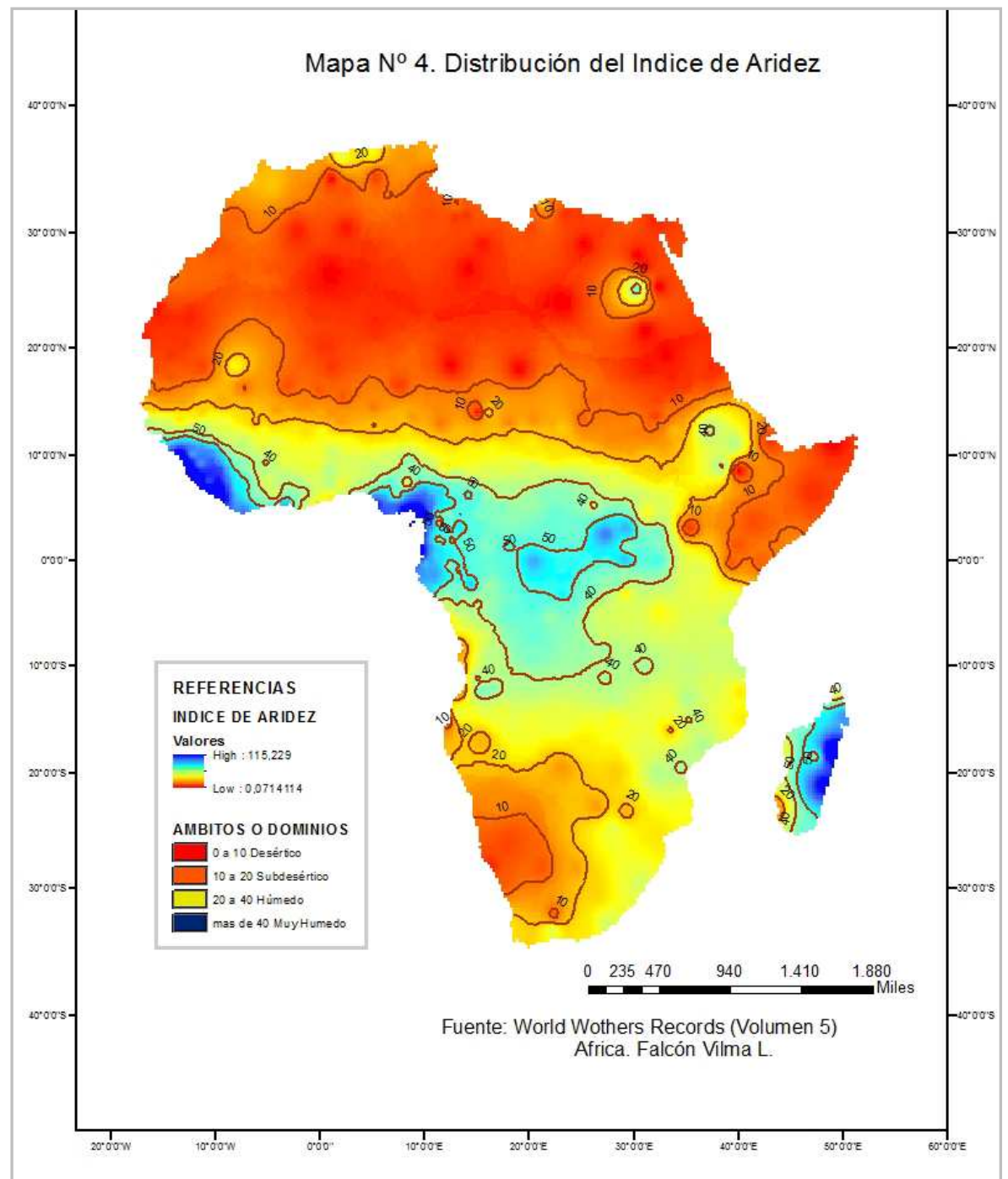

Esta actividad apunta a responder algunas preguntas como: ¿qué reflejan los valores del Índice de Aridez?,¿cómo se distribuyen los valores en el continente africano?. Cuando se afirma que en África las isotermas e isohietas presentan un trazado casi zonal con marcado paralelismo, ¿puede afirmarse lo mismo para este indicador? ¿Puede afirmarse que al Sur del Ecuador se presenta la misma disposición que en el Norte?. Al responder estas sencillas preguntas el alumno se ve en

Publicado en formato digital: Mgtr. Vilma Lilian Falcón. APLICACIONES DIDACTICAS CON SISTEMAS DE INFORMACIÓN GEOGRÁFICA. UNA APROXIMACIÓN AL CLIMA Y EL PAISAJE RESULTANTE EN EL CONTINENTE AFRICANO. Resúmenes. Revista Geográfica Digital. IGUNNE. Facultad de Humanidades. UNNE. Año 11. № 21. Enero - Junio 2014. ISSN 1668-5180 Resistencia, Chaco.

En: http://hum.unne.edu.ar/revistas/geoweb/default.htm 
Revista Geográfica Digital. IGUNNE. Facultad de Humanidades. UNNE. Año 11. № 21.

Enero - Junio 2014. ISSN 1668-5180 Resistencia, Chaco

la necesidad de complementar saberes previos con los nuevos contenidos desarrollados y estudiados en las clases teóricas.

La utilidad de incorporar al mapa las isolíneas de 10, 20 y 40 permite definir en forma aproximada la distribución de las áreas definidas anteriormente, secas o húmedas en el continente y puede constituirse en un insumo para explicar la distribución general de las formaciones vegetales, aunque para un estudio más pormenorizado el alumno deberá tener en cuenta otros aspectos, ya se trate del comportamiento general de la atmósfera como de la influencia de otros elementos locales como por ejemplo el relieve, la proximidad o lejanía al mar, entre otros.

Conviene recordar que este cálculo forma parte del balance hídrico y su resultado, como lo señala Bruniard, E. (1992:40), aún cuando fue concebido para explicar las condiciones del escurrimiento superficial, al observar su distribución geográfica permite advertir las relaciones generales con las formas de vegetación natural. En este sentido, el autor distingue las siguientes asociaciones:

\begin{tabular}{|c|l|l|}
\hline \multicolumn{2}{|c|}{ Cuadro No 1 } \\
\hline Valor del IA & \multicolumn{1}{|c|}{ Ámbito } & \multicolumn{1}{c|}{ Características } \\
\hline 0 a 10 & Desértico & $\begin{array}{l}\text { Suelos desnudos o con hierbas y } \\
\text { arbustos dispersos, sin } \\
\text { escurrimiento superficial y con } \\
\text { exigencias para cualquier cultivo. }\end{array}$ \\
\hline 10 a 20 & Subdesértico & $\begin{array}{l}\text { Estepas y sabanas (hierbas, } \\
\text { arbustos y algunos árboles } \\
\text { espinosos) con escurrimiento } \\
\text { temporario y posibilidad de } \\
\text { practicar cultivos de secano. }\end{array}$ \\
\hline 20 a 40 & Húmedo & $\begin{array}{l}\text { Praderas y parques, con } \\
\text { escurrimiento externo }\end{array}$ \\
\hline Superior a 40 & Muy Húmedo & $\begin{array}{l}\text { Predominio de bosques y } \\
\text { abundantes excedentes hídricos }\end{array}$ \\
\hline
\end{tabular}

Se parte de considerar que las adaptaciones morfológicas de las plantas dependen del proceso generado por los aportes pluviométricos, la utilización de la humedad por los vegetales, la evapotranspiración y la evaporación física del suelo. Esta asociación de elementos puede ayudar observar la distribución espacial del indicador de humedad-sequedad en el Espacio estudiado y al mismo tiempo abre las puertas a la búsqueda de la explicación de las causas y consecuencias de esa distribución

Publicado en formato digital: Mgtr. Vilma Lilian Falcón. APLICACIONES DIDACTICAS CON SISTEMAS DE INFORMACIÓN GEOGRÁFICA. UNA APROXIMACIÓN AL CLIMA Y EL PAISAJE RESULTANTE EN EL CONTINENTE AFRICANO. Resúmenes. Revista Geográfica Digital. IGUNNE. Facultad de Humanidades. UNNE. Año 11. № 21. Enero - Junio 2014. ISSN 1668-5180 Resistencia, Chaco. 
requiriendo para ello establecer las correlaciones necesarias entre las variables naturales.

\section{Comparación y comprobación de los resultados}

Es posible aplicar el principio geográfico de comparación y para ello se propone al estudiante observar otras cartografías, próximas o referidas a las mismas temáticas trabajadas, realizadas por otros autores u organismos especializados y que servirán, por un lado, para cotejar la información resultante de las variables analizadas con las asociaciones y distribuciones propuestas por otros autores especializados en el tema y , por otra parte, para confirmar que los procedimientos y técnicas aplicados para la obtención de las representaciones fueron apropiados ó, si guardan diferencias sustanciales, reverlos nuevamente.

Para el primer caso y como un ejemplo de esta propuesta hemos seleccionado, de entre la diversidad de material cartográfico que pueden obtenerse de sitios oficiales en internet, un trabajo producido por el Departamento de Montes de la FAO ${ }^{2}$ (Food and Agriculture Organization por sus siglas en Inglés) que se encuadra muy apropiadamente en el aspecto que se trabaja en esta actividad práctica de la cátedra. A modo ilustrativo se muestran las cartografías a comparar.
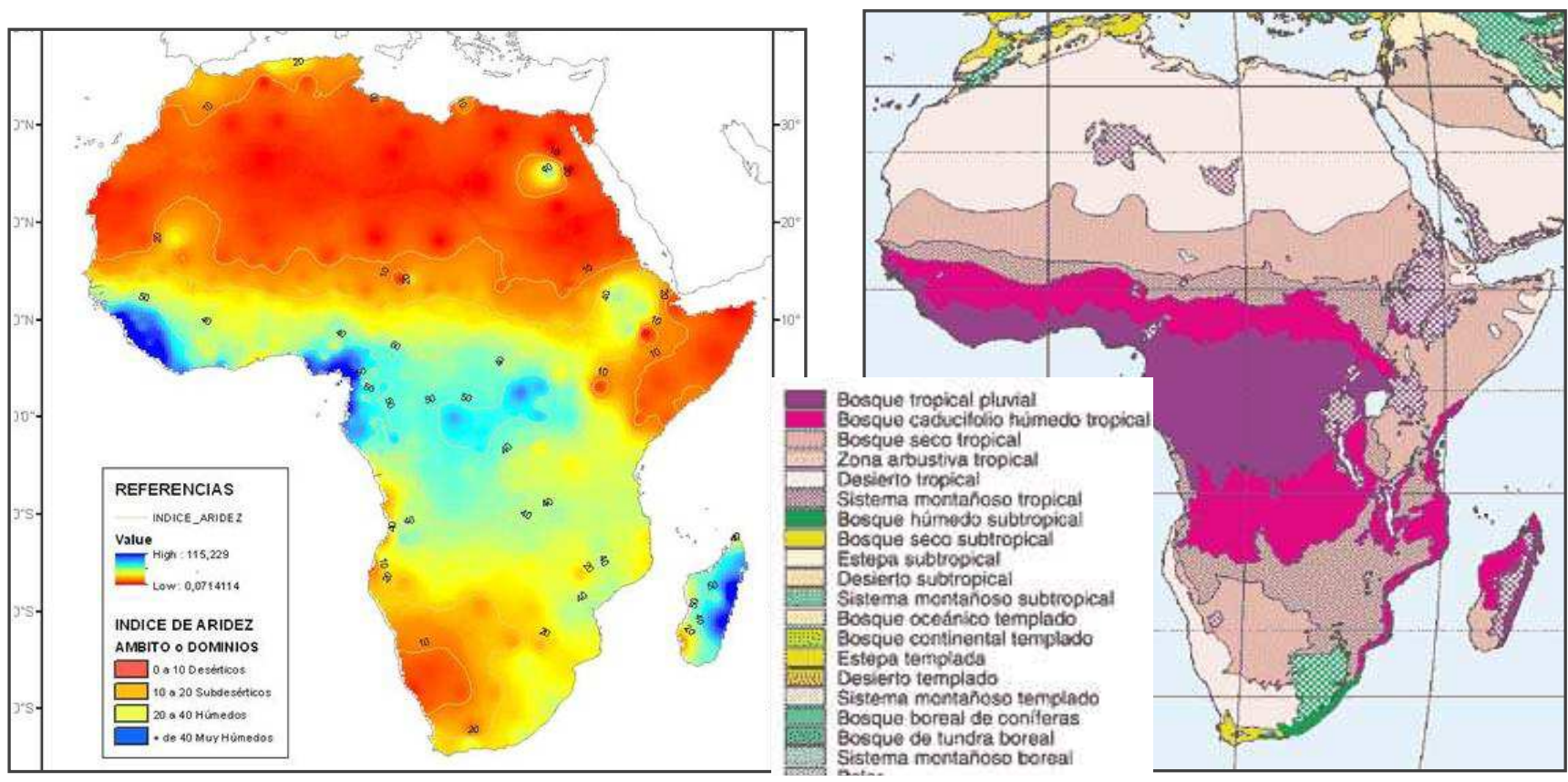

${ }^{2}$ Se trata del Documento Evaluación de los recursos forestales mundiales 2000, Producido por el Departamento de Montes y que se encuentra disponible en el repositorio de Documentos del la FAO en el sitio: http://www.fao.org/docrep/005/y1997s/y1997s0j.htm\#TopOfPage.

Publicado en formato digital: Mgtr. Vilma Lilian Falcón. APLICACIONES DIDACTICAS CON SISTEMAS DE INFORMACIÓN GEOGRÁFICA. UNA APROXIMACIÓN AL CLIMA Y EL PAISAJE RESULTANTE EN EL CONTINENTE AFRICANO. Resúmenes. Revista Geográfica Digital. IGUNNE. Facultad de Humanidades. UNNE. Año 11. № 21. Enero - Junio 2014. ISSN 1668-5180 Resistencia, Chaco.

En: http://hum.unne.edu.ar/revistas/geoweb/default.htm 
Revista Geográfica Digital. IGUNNE. Facultad de Humanidades. UNNE. Año 11. № 21.

Enero - Junio 2014. ISSN 1668-5180 Resistencia, Chaco

Tanto la información representada en el mapa "Zonas ecológicas de Africa" como la caracterización sintética de cada una de ellas permiten al alumno establecer las debidas comparaciones entre los ámbitos o dominios obtenidos con la representación del Índice de Aridez y las características de las zonas descriptas en el trabajo de la FAO.

Es así que se puede analizar y ampliar la información de cada ámbito descripto en el Cuadro № 1, tal como se muestra a continuación.

\begin{tabular}{|c|c|c|c|}
\hline \multicolumn{4}{|c|}{ Cuadro № 2: Ejemplo del análisis comparativo } \\
\hline \multicolumn{3}{|c|}{ INDICE DE ARIDEZ } & ZONAS ECOLOGICAS \\
\hline Valor & Ámbito & $\begin{array}{c}\text { Características } \\
\text { (Bruniard, E. 1992) }\end{array}$ & $\begin{array}{c}\text { Descripción } \\
\text { Departamento de Montes (FAO) }\end{array}$ \\
\hline 10 a 20 & Subdesértico & $\begin{array}{l}\text { Estepas y sabanas } \\
\text { (hierbas, arbustos y } \\
\text { algunos árboles } \\
\text { espinosos) con } \\
\text { escurrimiento } \\
\text { temporario y } \\
\text { posibilidad de } \\
\text { practicar cultivos de } \\
\text { secano. }\end{array}$ & $\begin{array}{l}\text { Zona Arbustiva Tropical } \\
\text { En la región saheliana, el desierto de Kalahari y la } \\
\text { parte sudoccidental de Madagascar, las lluvias } \\
\text { disminuyen, aunque las temperaturas siguen siendo } \\
\text { elevadas. El régimen de precipitaciones nunca alcanza } \\
\text { los } 1000 \mathrm{~mm} \text { y muy rara vez llega a } 200 \mathrm{~mm} \text { en las } \\
\text { zonas más secas. (...)En esas zonas, muy secas, por lo } \\
\text { general se dan en forma espontánea seudoestepas y } \\
\text { matorrales. En el sur de la zona del Sahel existen } \\
\text { praderas arboladas (sobre todo con las especies } \\
\text { Anogeissus y Acacia), y en el norte praderas } \\
\text { semidesértica }\end{array}$ \\
\hline 20 a 40 & Húmedo & $\begin{array}{lr}\text { Praderas } & y \\
\text { parques, } & \text { con } \\
\text { escurrimiento } & \\
\text { externo } & \end{array}$ & $\begin{array}{l}\text { Bosque Caducifolio Húmedo Tropical } \\
\text { (....) La estación seca es pronunciada, y puede durar } \\
\text { hasta seis meses. Existe una única estación de las } \\
\text { lluvias, en verano, aunque se observan marcadas } \\
\text { variaciones de acuerdo con las regiones. ...... } \\
\text { Bosques Secos Tropicales } \\
\text { (...)En este contexto de mayor aridez, el tipo de } \\
\text { vegetación dominante es el bosque abierto. En la } \\
\text { región zambiana se encuentran formaciones de } \\
\text { miombo, más seco, bosques de mopane } \\
\text { (Colophospermum mopane) o montes sudaneses en } \\
\text { los valles y depresiones meridionales, y matorrales en }\end{array}$ \\
\hline
\end{tabular}

\footnotetext{
${ }^{3}$ Fuente: Zonas Ecológicas de Africa (Fig. 1.12). Departamento de Montes. FAO. En: http://www.fao.org/docrep/005/y1997s/y1997s13.jpg
}

Publicado en formato digital: Mgtr. Vilma Lilian Falcón. APLICACIONES DIDACTICAS CON SISTEMAS DE INFORMACIÓN GEOGRÁFICA. UNA APROXIMACIÓN AL CLIMA Y EL PAISAJE RESULTANTE EN EL CONTINENTE AFRICANO. Resúmenes. Revista Geográfica Digital. IGUNNE. Facultad de Humanidades. UNNE. Año 11. № 21. Enero - Junio 2014. ISSN 1668-5180 Resistencia, Chaco.

En: http://hum.unne.edu.ar/revistas/geoweb/default.htm 
Revista Geográfica Digital. IGUNNE. Facultad de Humanidades. UNNE. Año 11. № 21.

Enero - Junio 2014. ISSN 1668-5180 Resistencia, Chaco

\begin{tabular}{|c|c|c|c|c|}
\hline & & & & $\begin{array}{l}\text { las tierras bajas meridionales, con ejemplares de } \\
\text { Acacia caffra, Acacia davyi y Acacia luederitzii. En la } \\
\text { región sudanesa...... }\end{array}$ \\
\hline $\begin{array}{c}\text { Superior } \\
\text { a } 40\end{array}$ & $\begin{array}{l}\text { Muy } \\
\text { Húmedo }\end{array}$ & $\begin{array}{l}\text { Predominio } \\
\text { bosques } \\
\text { abundantes } \\
\text { excedentes } \\
\text { hídricos }\end{array}$ & $\begin{array}{r}\text { de } \\
y\end{array}$ & $\begin{array}{l}\text { Bosque Tropical Pluvial } \\
\text { Esta zona abarca la parte central de África a uno y } \\
\text { otro lado del ecuador así como la costa sudoriental. } \\
\text { La formación de mayor extensión es el bosque } \\
\text { húmedo de tierras bajas guineo-congoleño, que se } \\
\text { concentra en la cuenca del Congo. Se trata de un } \\
\text { bosque alto y tupido con una altura superior a } 30 \mathrm{~m} \text { y } \\
\text { árboles emergentes de hasta } 60 \mathrm{~m} \text { y diferentes } \\
\text { estratos. Algunas especies son caducifolias pero el } \\
\text { bosque en sí es perenne o semiperenne......... }\end{array}$ \\
\hline
\end{tabular}

\section{Perfiles Comparativos}

Siguiendo con la propuesta realizada por Bruniard, E. (1992:42-43) pueden realizarse perfiles comparativos para destacar la correspondencia espacial entre los elementos analizados y representados. En este sentido, si a los dos mapas anteriores (Mapas 3 y 4) se agregan las representaciones que muestran la distribución de las temperaturas y precipitaciones en el continente, realizando algunas líneas de cortes pueden ensayarse descripciones sintéticas con las asociaciones de las diferentes áreas o zonas interceptadas por el corte.

Para realizar un Grafico de perfil se utiliza la barra de herramientas interactiva de ArcGIS (en la versión 10.1) 3D Analyst. Los perfiles que se generaron fueron realizados a partir de una superficie ráster que, en este caso particular, correspondieron a las cartografías obtenidas utilizando el método de interpolación IDW en Spatial Analisis Tools.

Publicado en formato digital: Mgtr. Vilma Lilian Falcón. APLICACIONES DIDACTICAS CON SISTEMAS DE INFORMACIÓN GEOGRÁFICA. UNA APROXIMACIÓN AL CLIMA Y EL PAISAJE RESULTANTE EN EL CONTINENTE AFRICANO. Resúmenes. Revista Geográfica Digital. IGUNNE. Facultad de Humanidades. UNNE. Año 11. № 21. Enero - Junio 2014. ISSN 1668-5180 Resistencia, Chaco.

En: http://hum.unne.edu.ar/revistas/geoweb/default.htm 
Revista Geográfica Digital. IGUNNE. Facultad de Humanidades. UNNE. Año 11. № 21.

Enero - Junio 2014. ISSN 1668-5180 Resistencia, Chaco
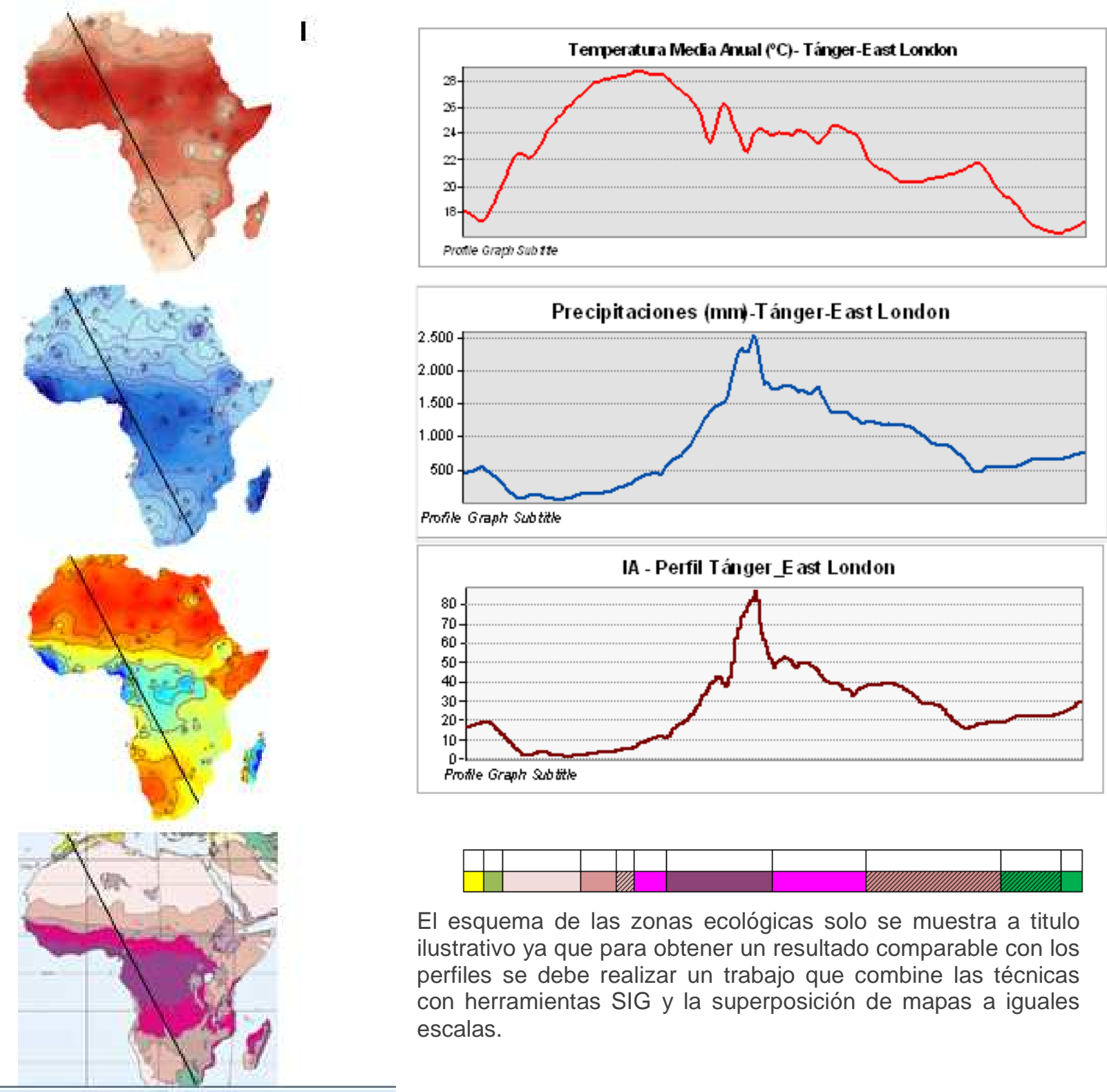

El esquema de las zonas ecológicas solo se muestra a titulo ilustrativo ya que para obtener un resultado comparable con los perfiles se debe realizar un trabajo que combine las técnicas con herramientas SIG y la superposición de mapas a iguales escalas.

\section{Conclusiones}

La experiencia que expusimos en este trabajo resume una gama muy amplia de posibilidades de uso de algunos recursos tecnológicos como los que proveen los Sistemas de Información Geográfica. Aún cuando se trató de un aspecto muy definido como lo fue el análisis de datos termo-pluviométricos de localidades de África, en el

Publicado en formato digital: Mgtr. Vilma Lilian Falcón. APLICACIONES DIDACTICAS CON SISTEMAS DE INFORMACIÓN GEOGRÁFICA. UNA APROXIMACIÓN AL CLIMA Y EL PAISAJE RESULTANTE EN EL CONTINENTE AFRICANO. Resúmenes. Revista Geográfica Digital. IGUNNE. Facultad de Humanidades. UNNE. Año 11. № 21. Enero - Junio 2014. ISSN 1668-5180 Resistencia, Chaco.

En: http://hum.unne.edu.ar/revistas/geoweb/default.htm 
Revista Geográfica Digital. IGUNNE. Facultad de Humanidades. UNNE. Año 11. № 21.

Enero - Junio 2014. ISSN 1668-5180 Resistencia, Chaco

proceso del trabajo fueron apareciendo diversas opciones de análisis conforme iban surgiendo las respuestas a las pregunta planteadas y a la vez generaban nuevos interrogantes que requerían ensayar otros procedimientos con los aplicativos del software elegido. Todo ello no viene más que a reforzar la idea de complementariedad entre recursos y conocimiento disciplinar, especialmente porque se recurre a conceptos y saberes previos de las dos áreas del conocimiento que se ponen en juego: lo geográfico como base disciplinar de la experiencia desde la cual se realizan las preguntas que dan origen al trabajo (¿cómo se manifiestan los componentes del medio natural en África?) y lo tecnológico no solo como una herramienta sino fundamentalmente como el eje alrededor del cual se fue construyendo el conocimiento en una suerte de retroalimentación positiva.

En el ámbito de la educación universitaria el uso de los recursos abre un enorme abanico de posibilidades para la práctica pedagógica y si a ello se suma la ciencia geográfica como marco de análisis y estudio, diríamos que estamos ante un contexto ideal de trabajo.

\section{Bibliografía}

- Bruniard, Enrique (1992). Climatología. Procesos y tipos climáticos. Colección Geográfica. Editorial CEYNE SRL. Buenos Aires.

- Falcón, Vilma y Da Silva, Cristian (2013). El uso de los SIG en la práctica pedagógica. Aportes para el estudio de África y Oceanía. En: Actas de: II Congreso nacional de Tecnologías de la Información Geográfica y II Jornadas de Sistemas de Información Geográfica. Universidad Nacional de General Sarmiento. Buenos Aires. 3 y 4 de Octubre de 2013.-

- GK Gonzalez Zumba (2006). Manual curso análisis espacial. En: http://dspace.ups.edu.ec/bitstream/123456789/204/3/Capitulo\%202.pdf

- Mendez, Ricardo y Molinero, Fernando (2002). Espacios y Sociedades. Introducción a la Geografía regional del mundo. 6ta. Edición. Editorial ARIEL, Barcelona.

- Murillo, Diego; Ortega, Irene; et. al. (2012). Comparación de Métodos de Interpolación para la generación de mapas de ruido en entornos urbano. En: web.usbmed.edu.co/usbmed/fing/v3n1/v3n1a7.pdf

- Rodolfo Franco (2008-2011). Ejemplo para generación de isolineas en Arcgis. Universidad Distrital FJC, Facultad del Medio Ambiente y Recursos Naturales . Bogota DC, Colombia

- World Weather Records (Volumen 5: Africa, 1968 y Volumen 6: Antarctica, Australia, Oceanics Islands and Ocean Weather Stations, 1968) Editado por U.S. Departament of Comerce. Washington.

- Zapetini, Maria Ceciilia (2007). Enseñanza de la Geografía e Informática. En: Geograficando, 2007 № 3 pag. 189-203. http://www.memoria.fahce.unlp.edu.ar/art_revistas/pr.3664/pr.3664.pdf.

Publicado en formato digital: Mgtr. Vilma Lilian Falcón. APLICACIONES DIDACTICAS CON SISTEMAS DE INFORMACIÓN GEOGRÁFICA. UNA APROXIMACIÓN AL CLIMA Y EL PAISAJE RESULTANTE EN EL CONTINENTE AFRICANO. Resúmenes. Revista Geográfica Digital. IGUNNE. Facultad de Humanidades. UNNE. Año 11. № 21. Enero - Junio 2014. ISSN 1668-5180 Resistencia, Chaco. 